\title{
Visualization of charge transport through Landau levels in graphene
}

\author{
G. Nazin ${ }^{1}$, Y. Zhang ${ }^{2}$, L. Zhang ${ }^{3}$, E. Sutter ${ }^{1}$ and P. Sutter ${ }^{1 \star}$
}

Band bending and the associated spatially inhomogeneous population of Landau levels play a central role in the physics of the quantum Hall effect (QHE) by constraining the pathways for charge-carrier transport and scattering ${ }^{1}$. Recent progress in understanding such effects in low-dimensional carrier gases in conventional semiconductors has been achieved by realspace mapping using local probes ${ }^{2,3}$. Here, we use spatially resolved photocurrent measurements in the QHE regime to study the correlation between the distribution of Landau levels and the macroscopic transport characteristics in graphene. Spatial maps show that the net photocurrent is determined by hot carriers transported to the periphery of the graphene channel, where QHE edge states provide efficient pathways for their extraction to the contacts. The photocurrent is sensitive to the local filling factor, which allows us to reconstruct the local charge density in the entire conducting channel of a graphene device.

Since the demonstration of the unusual half-integer QHE in graphene ${ }^{4,5}$, many related QHE experiments have been interpreted within the framework of edge-state transport ${ }^{6-8}$, that is, the backscattering-free flow of charge through edge states ${ }^{9}$ bounded by insulating barriers with incompressible electron densities ${ }^{10}$. Although compressible and incompressible electron densities have recently been observed in graphene ${ }^{11}$, their role in shaping the QHE in graphene remains to be explained. Spatially inhomogeneous charge distributions owing to adsorbate-induced surface doping ${ }^{12}$ are expected to be particularly pronounced in graphene and could cause deviations from pure edge-state transport. Here, we use scanning photocurrent microscopy to explore these effects by mapping carrier propagation through graphene Landau levels in the QHE regime.

The experiments were carried out on two-terminal monolayer graphene field-effect devices at $4.2 \mathrm{~K}$ and in magnetic fields $B$ up to $\pm 9 \mathrm{~T}$ (Fig. 1a,b). In these conditions, the conductance of our devices (Fig. 1c) shows series of local extrema ${ }^{6,8,11,13,14}$ associated with individual Landau levels ${ }^{13,15}$, with maxima predicted to occur at quantized Hall conductances of 2, 4, 6, 10 and $14 e^{2} / h$ (refs 13,15). The observed maxima are higher owing to an inhomogeneous filling-factor distribution across the device, as shown below.

Photocurrent maps were obtained by scanning a focused laser across the graphene channel, and recording the two-terminal photocurrent signal as a function of beam position. Previously, a similar approach has been used to investigate contact-induced band bending ${ }^{16-19}$ and the photo-thermoelectric effect ${ }^{20}$ in graphene at $B=0$, as well as the electrostatics of the QHE in conventional semiconductor devices ${ }^{21-23}$. We observe that the gate-voltagedependent photocurrent at fixed locations is oscillatory, with polarity determined by the direction of the magnetic field (Fig. 1d).
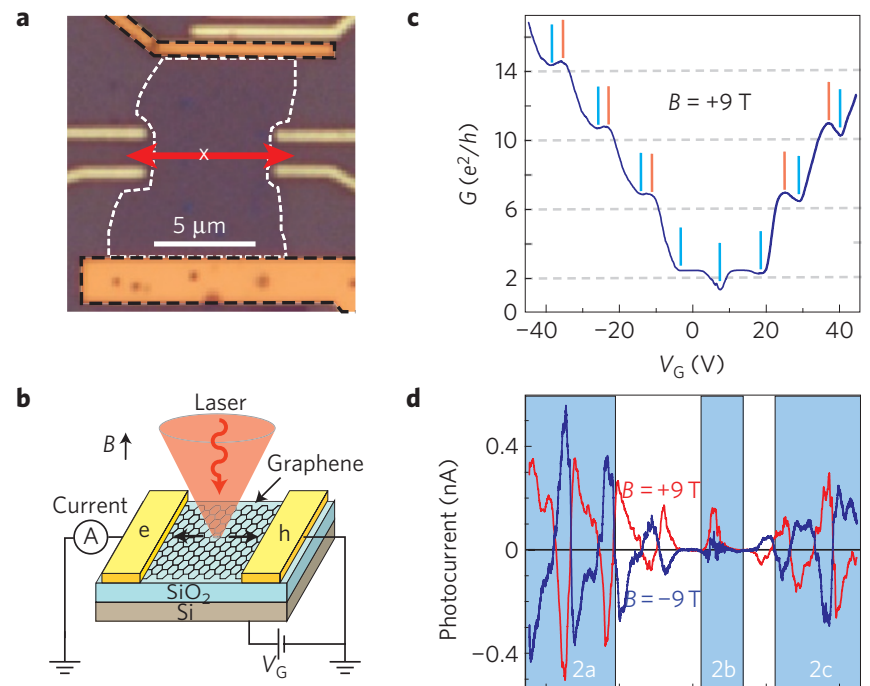

d

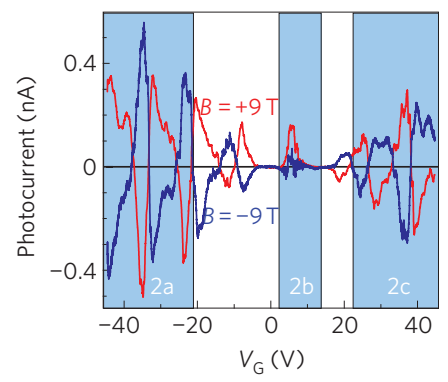

Figure 1 | Photocurrent measurements on graphene devices. a, Optical image of the device. The dashed outlines mark the channel and the two connected electrodes. The four side electrodes are not in contact with the device. b, Schematic of the photocurrent measurement. c, Conventional two-terminal conductivity, $G$, as a function of gate voltage, $V_{G}$. The blue and red lines mark local conductivity minima and maxima. d, Photocurrent measured with the laser spot at the centre of the graphene channel (cross in a) for $B= \pm 9 \mathrm{~T}$. The shaded regions denoted $2 \mathrm{a}-2 \mathrm{c}$ mark the different $V_{\mathrm{G}}$ ranges in the sequence of photocurrent maps of Fig. 2.

Such local oscillations are due to a recurring global photocurrent distribution across the device, synchronous with the filling of consecutive Landau levels (Fig. 2). Although the patterns for nand p-type doping and near the neutrality point differ, pairs of maps in the same columns of Fig. 2a,c show nearly identical spatial photocurrent distributions. For specific carrier densities, such as $V_{\mathrm{G}}=-45 \mathrm{~V},-37.5 \mathrm{~V},-33 \mathrm{~V},-25.5 \mathrm{~V}$ and so on, we observe, for instance, extended, sharply delineated areas with photocurrent response of the same polarity aligned roughly along the channel axis.

To further analyse these periodically recurring photocurrent distributions, we consider the photocurrent as a function of gate voltage along a line across the graphene channel (Fig. 3a). In a perpendicular magnetic field, the energy-level spectrum of Dirac fermions in graphene comprises n- and p-type Landau levels, as well as a degenerate level $n=0$ at the neutrality point ${ }^{5}$ (Fig. $3 \mathrm{~b}$ ). In the

\footnotetext{
${ }^{1}$ Center for Functional Nanomaterials, Brookhaven National Laboratory, Upton, New York 11973, USA, ${ }^{2}$ Department of Physics and Astronomy, Stony Brook University, Stony Brook, New York 11794, USA, ${ }^{3}$ Condensed Matter Physics and Materials Science Department, Brookhaven National Laboratory, Upton, New York 11973, USA. *e-mail: psutter@bnl.gov.
} 
a
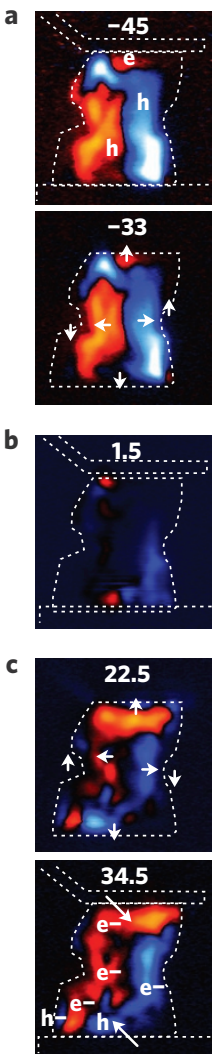

$10 \mu \mathrm{m}$
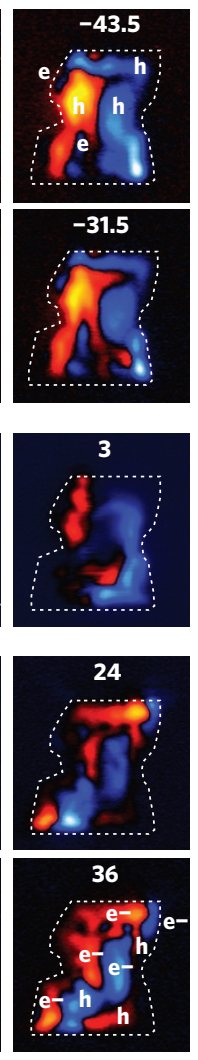
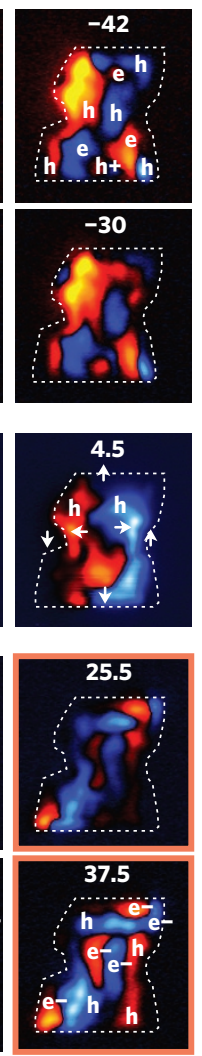

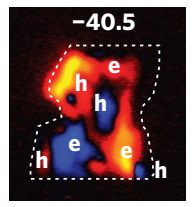

$-28.5$
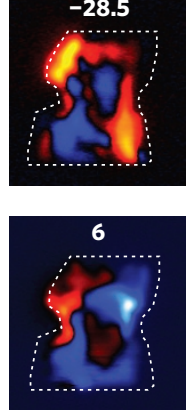

27

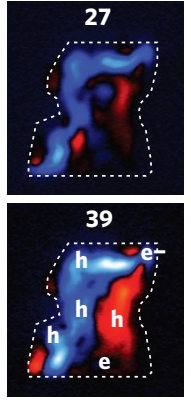

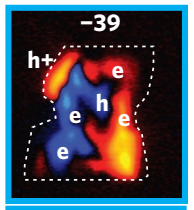
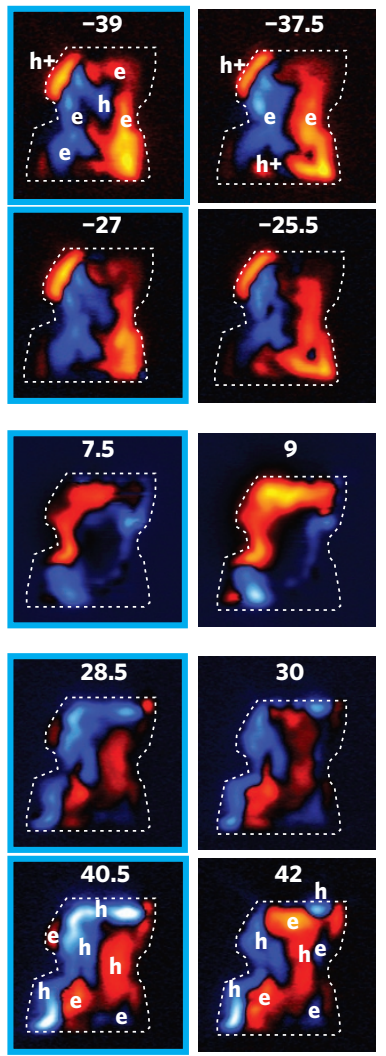
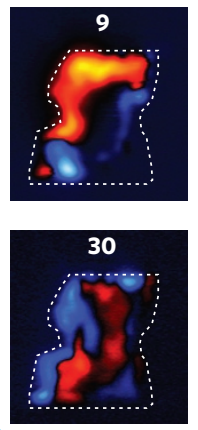

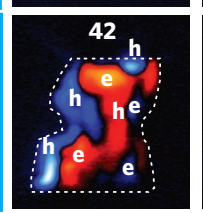

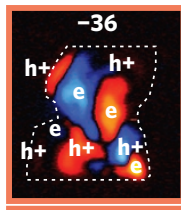
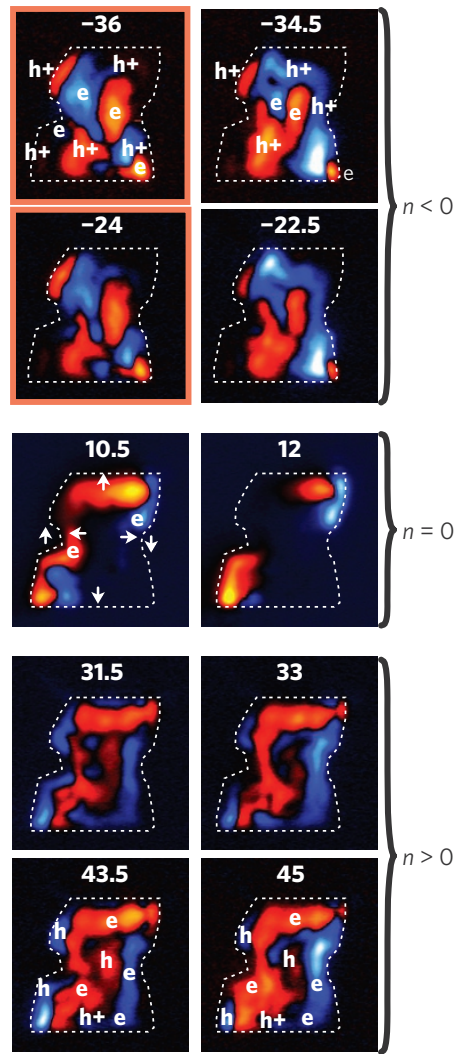

33

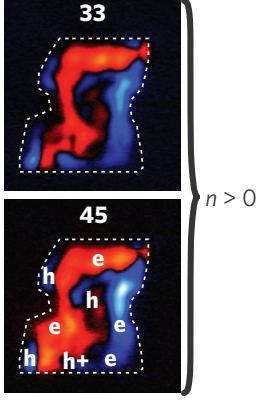

$(n A)$

Figure 2 | Spatial photocurrent maps for different gate voltages at $\mathbf{B}=\mathbf{9}$ T. a, Photocurrent maps of $p$-type Landau levels. $\mathbf{b}$, Photocurrent maps near the $n=0$ Landau level. c, Photocurrent maps of n-type Landau levels. Positive current corresponds to hole collection into the bottom contact of the device

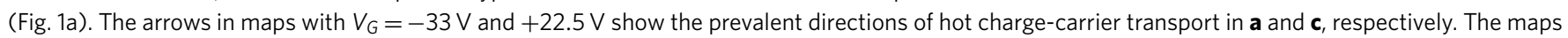
with blue (red) borders correspond to conductance minima (maxima) in Fig. 1b. In the top row of $\mathbf{a}$ and the bottom row of $\mathbf{c}$, the predominant type of hot

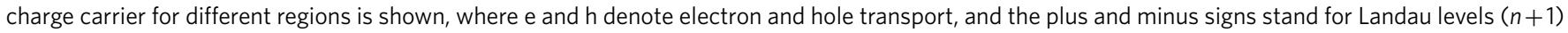

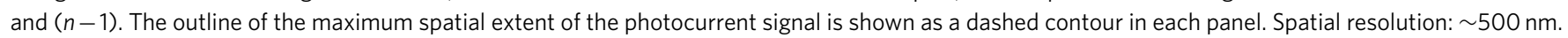
Laser wavelength/power: $650 \mathrm{~nm} / 160 \mathrm{nW}$.

photocurrent section of Fig. 3a, every Landau level except $n=0$ is associated with a distinct 'butterfly' pattern comprising four lobes of alternating polarity. The sign of the photocurrent response changes abruptly on crossing the channel centreline, and on either side alternates with varying $V_{\mathrm{G}}$. The patterns suggest that the energies of Landau levels are higher in the centre than at the edges. This type of band bending has been observed in all tested devices, and similar band bending is probably present to some degree in all graphene devices used in conventional transport experiments.

The butterfly patterns of Fig. 3a can be understood by considering photocurrent generation and collection in graphene in the QHE regime (Fig. 3c,d). At our photon energy, local light absorption excites an electron-hole pair to high-order Landau levels, followed by rapid relaxation and either recombination or charge transport. Owing to screening of the electrical fields in the interior of the channel, the sensitivity of the photocurrent to the magnetic field (Fig. 1d) implies that carriers generated in the interior can reach the edges to be extracted to the contacts by edgestate transport (see Supplementary Information). For the same carrier type, the currents carried at opposing edges are anti-parallel, with directions given by the vector product of the $B$ field and the gradient of the Landau-level edge-state band bending. Carriers that relax to the partially filled Landau level at the Fermi energy, $E_{\mathrm{F}}$, can propagate over long distances through the percolating conducting network formed by this Landau level in the presence of disorder, that is, are extracted symmetrically at both edges and give zero net contribution to the photocurrent. In contrast, intralevel relaxation of 'hot' electrons (holes) in Landau levels not aligned with $E_{\mathrm{F}}$ leads to transport involving states at the bottom (top) of the disorderbroadened levels, where localization effects are stronger compared with the Landau level at $E_{\mathrm{F}}$ (ref. 11). The net photocurrent owing to these carriers is dominated by the edge closest to the point of illumination. This physical picture is analogous to the microscopy of photo- and electron-beam-induced current in semiconductor devices $^{24,25}$, where the net induced current reflects the transport of minority carriers. In the present case, both hot electrons and hot holes simultaneously act as 'minority' carriers. The polarity of the net photocurrent is determined by the type of hot carriers reaching the edges in larger numbers, which in turn depends on the local filling factor $v$ controlling the phase space available for relaxation to $E_{\mathrm{F}}$. Indeed, for a nearly empty Landau level at $E_{\mathrm{F}}$, the relaxation of hot electrons to this Landau level must be more efficient than that of holes, so that the latter prevail in the hot-carrier transport. Conversely, for a nearly full Landau level at $E_{\mathrm{F}}$ hot electrons will dominate the net photocurrent. For high-order Landau levels, the transitions between 'mostly hot-electron' and 'mostly hot-hole' transport should occur roughly at zero- and at half-filling of the individual Landau levels.

Using this picture of photocurrent generation, we can explain the observed characteristic butterfly patterns. The schematics of Fig. 4 


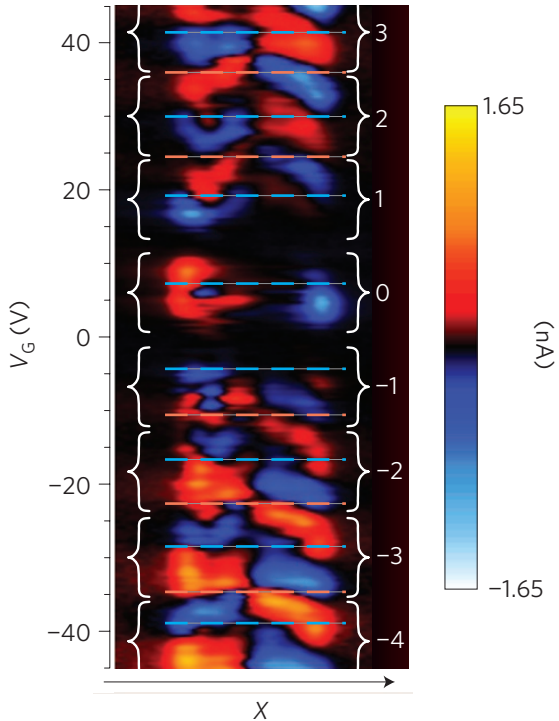

b

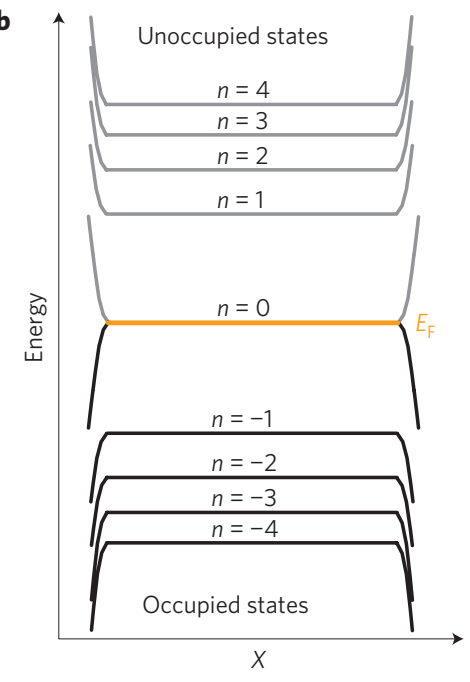

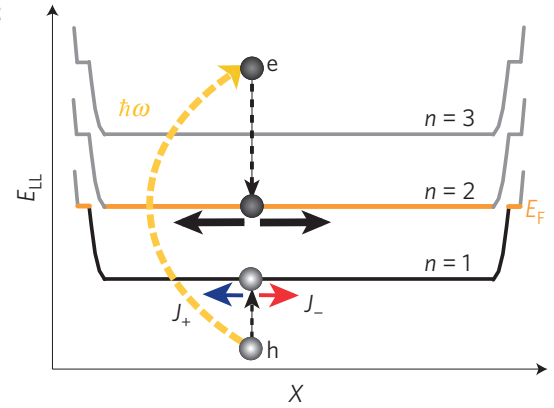

d

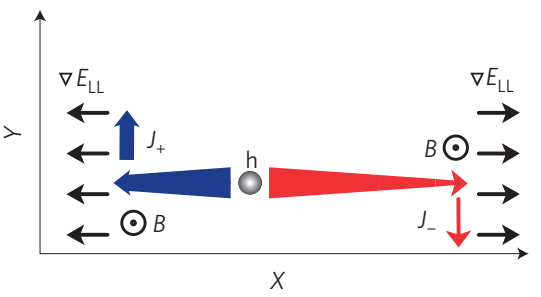

Figure $3 \mid V_{G}$ dependence of photocurrent across the device, and photocurrent-generation mechanism. $a$, Photocurrent as a function of gate voltage along the arrow shown in Fig. 1a, extracted from 240 individual scans across the graphene channel for $V_{\mathrm{G}}$ from $-45 \mathrm{~V}$ to $+45 \mathrm{~V}(B=9 \mathrm{~T})$. The dashed blue and red lines correspond to conductivity minima and maxima in Fig. $1 \mathrm{~b}$. The braces indicate the $V_{\mathrm{G}}$ ranges corresponding to individual Landau levels, numbered sequentially $n=0, \pm 1, \pm 2$ and so on. $\mathbf{b}$, Landau levels in graphene. At the edges, the magnetic field bends levels with $n>0$ ( $n<0$ ) upward (downward); the degenerate $n=0$ level splits into two branches. c,d, Illustration of photocurrent generation and collection, assuming a partially occupied $n=2$ Landau level. The level with $n=1$ is fully occupied except at the edges where it forms a set of edge states. c, Light absorption and electron-hole pair excitation. $\mathbf{d}$, Collection of hot carriers involving initial slow transport across the channel ( $X$ direction) and subsequent fast transport through edge states ( $Y$ direction). The arrow thicknesses indicate variations in current owing to carrier relaxation to the Fermi level.

illustrate the spatial photocurrent distribution for two situations (at different $V_{\mathrm{G}}$ ): one Landau level close to half-filling in the interior of the channel (Fig. 4a-n-type Landau level; Fig. 4ep-type Landau level); and two Landau levels close to half-filling (Fig. 4b-n-type Landau levels; Fig. $4 \mathrm{f}-\mathrm{p}$-type Landau levels). This picture is analogous to the one developed in ref. 26. If, for instance, the $n=2$ level forms a compressible, partially filled state spanning nearly the entire width of the channel, the charge density would rearrange itself to screen external potentials and disorder, leading to a non-uniform population of this Landau level across the device ${ }^{11}$ (Fig. 4a). According to the discussion in the previous paragraph, electrons dominate the photocurrent when $v_{2}>v_{2}{ }^{0}$; holes prevail in areas with $v_{2}<v_{2}{ }^{0}$, where $v_{2}{ }^{0}$ corresponds to the filling factor for which electron and hole hot-carrier currents exactly compensate each other. This scenario indeed gives rise to a four-lobe pattern similar to that of Fig. 3a. One can readily generalize to a situation in which two n-type Landau levels intersect $E_{\mathrm{F}}$ in the interior (Fig. 4b), and to p-type Landau levels with downward edge band bending (Fig. 4e,f), all of which will give rise to similar butterfly patterns in the photocurrent collection. The $n=0$ Landau level at the neutrality point is an interesting special case. Here, Fig. 3 shows only two lobes in the photocurrent signal. In this regime, both the carrier type and band bending invert as the filling factor changes from $v<0$ to $v>0$; that is, sweeping $V_{\mathrm{G}}$ through the neutrality point does not alter the photocurrent polarity (Fig. $4 \mathrm{~d}, \mathrm{~h}$ ). The net hot-carrier current is always given by extraction to the nearest edge channel, producing a two-lobe pattern around the centreline of the device.

By tracing the evolution of the photocurrent signal with $V_{\mathrm{G}}$ in Fig. 3a, we can now identify the type of hot carriers giving rise to the net photocurrent response in every part of the device. We find that photocurrent maps in Fig. 2a,c corresponding to conductance minima involve a single Landau level close to halffilling over most of the device (Fig. 4a,e). Maps at the maxima show two different Landau levels at $E_{\mathrm{F}}$ in the centre and at the periphery (Fig. 4b,f); the dividing boundaries, traced in the zoomed experimental maps of Fig. 4c,g, are expected to be incompressible ${ }^{11}$, that is, form insulating barriers that profoundly affect the electrostatic landscape and current pathways in the device. In the conventional QHE, incompressible boundaries isolate counterpropagating currents in edge states ${ }^{2,3}$, reduce backscattering ${ }^{27}$, and hence give rise to conductance quantization. Similarly, they affect the magnetoconductance in graphene channels with non-uniform potential. For n-type Landau levels (Fig. $4 a-c)$, the conductance in a graphene channel with inhomogeneous charge distribution not only comprises contributions of edge states, but also includes the bulk conductance of a Landau level near the periphery (areas marked h in Fig. 4c), which also couples to the contacts. This bulk contribution causes the overall conductance to exceed the expected quantized values. For p-type Landau levels, Fig. 3a shows that the induced positive charge density is always highest in the centre of the device. The Landau-level crossing $E_{\mathrm{F}}$ inside the channel does not form edge states (Fig. $4 \mathrm{e}-\mathrm{g}$ ), but it can suppress backscattering between edge states at opposite sides of the channel. The reduced backscattering together with the bulk conductance of this Landau level (through areas marked e in Fig. $4 \mathrm{~g}$ ) again causes $G$ maxima higher than the expected quantized conductance values for this branch of the conductance curve.

The unique geometry of a two-dimensional carrier gas confined close to the surface makes graphene particularly suitable for scanning probe experiments. The advantage of the presented photocurrent-based approach is the capability to map not only the charge density, which could be determined by other scanning probe methods, but also the carrier propagation in graphene in the QHE regime. On the basis of the analysis of the photocurrent-collection mechanism in graphene subjected to high magnetic fields, our initial results demonstrate the formation of counter-propagating edge states, with direction of propagation determined by the unique Landau-level spectrum of graphene and the Landau-level bending at the edges of the channel. Furthermore, we observe the interplay between bulk and edge-state conduction owing to inhomogeneously populated Landau levels, a situation commonly found in graphene devices that gives rise to deviations from exact 
a

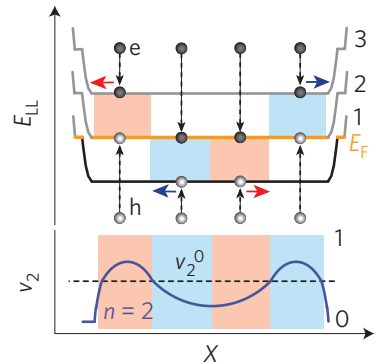

b
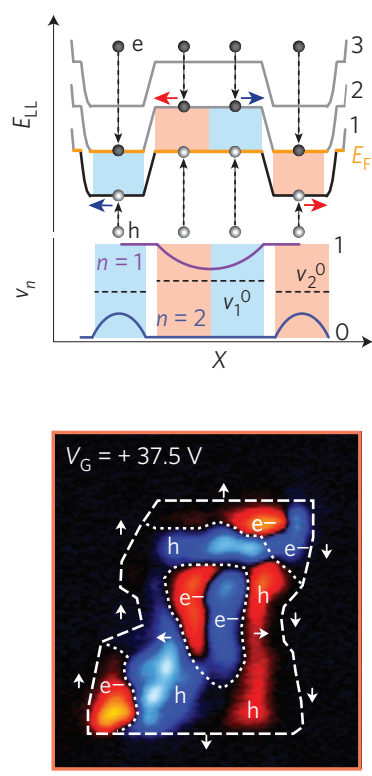

d

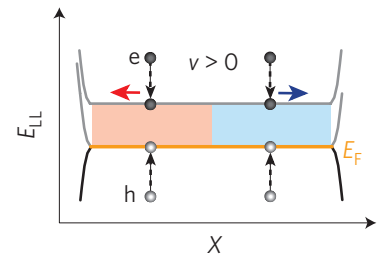

e

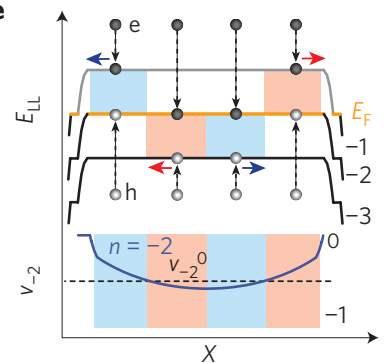

f

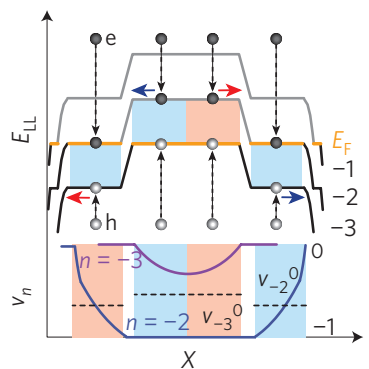

g

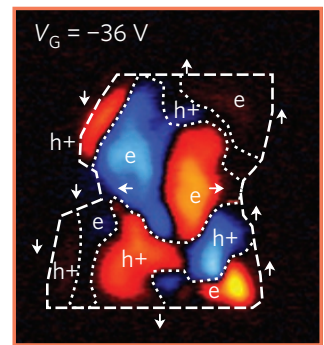

h

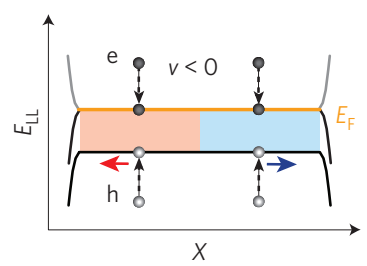

Figure 4 | Carrier relaxation and photocurrent collection in graphene devices in the QHE regime. a-d, Photocurrent collection for n-type doping. a, Dominant pathways of charge-carrier relaxation and collection across the graphene channel, for the case of one Landau level (here, $n=2$ ) near $E_{F}$ inside the channel; and local filling factor $v_{2}$ of this level across the device. Here and in all other panels, $v_{n}{ }^{0}$ corresponds to the filling factor (of level $n$ ) for which electron and hole hot-carrier currents exactly compensate each other. The coloured rectangles connect the Fermi level with the Landau levels responsible for hot-carrier transport; the colour indicates the resulting photocurrent polarity. $\mathbf{b}$. The same as in $\mathbf{a}$ for the case of two Landau levels $(n=1,2)$ at $E_{\mathrm{F}}$ inside the channel, and local filling factors of these levels across the device. Schematics are not to scale. c, Photocurrent map at $V_{G}=+37.5 \mathrm{~V}$. The approximate locations of the incompressible boundaries are shown as dotted contours. d, Carrier relaxation and collection for the $n=0$ Landau level near the neutrality point. e-h, Photocurrent collection for p-type doping. e, One Landau level $(n=-2)$ at $E_{\mathrm{F}}$, and local filling factor $v_{-2}$ of this level across the device. f, Two Landau levels at $E_{\mathrm{F}}(n=-2,-3)$ inside the channel, and local filling factors of these levels across the device. Schematics are not to scale. g, Photocurrent map at $V_{\mathrm{G}}=-36 \mathrm{~V}$. h, Carrier relaxation and collection for the $n=0$ Landau level.

conductance quantization. The present approach is well suited for more detailed investigations of the QHE physics in graphene,

including electrostatics and current pathways in externally biased devices and the effects of local disorder and contact-induced band bending. Finally, our method could be used to probe carrier transport and the QHE in high-mobility suspended graphene devices $^{28}$, giving access to phenomena that cannot be measured by conventional two-probe magnetotransport experiments.

\section{Methods}

Sample preparation. Monolayer graphene flakes for device fabrication were prepared on degenerately doped $\mathrm{Si}$ wafers with $300 \mathrm{~nm}$ thermal $\mathrm{SiO}_{2}$ using the 'Scotch-tape technique' similar to refs 29,30. Electrical contacts to chosen graphene flakes were defined using electron-beam lithography and deposition of $3 \mathrm{~nm}$ Ti and $30 \mathrm{~nm} \mathrm{Au}$. The devices were then vacuum-annealed at $120^{\circ} \mathrm{C}$ below $10^{-7} \mathrm{mbar}$, and transferred to the cryostat for phototransport measurements. Thus, prepared devices typically showed the neutrality point at gate voltages below $10 \mathrm{~V}$.

Photocurrent measurements. Electrical transport and phototransport experiments were carried out at $4.2 \mathrm{~K}$ in a ${ }^{4} \mathrm{He}$ bath cryostat with a variable-temperature insert, equipped with a superconducting magnet and an integrated confocal optical microscope used to project a focused laser beam onto the graphene channel while measuring the electrical response between the source and drain electrodes (top and bottom contacts in Fig. 1a) of the device; the side electrodes shown in Fig. la were not in contact with the device channel. Owing to the low resistance of graphene devices, measurements of photovoltage were more appropriate than photocurrent for low-level signals. To avoid large signal-level variations between photovoltage maps, we report photocurrent $(\mathrm{PC})$ data converted from photovoltage (PV) measurements using $\mathrm{PC}\left(V_{\mathrm{G}}\right)=\mathrm{PV}\left(V_{\mathrm{G}}\right) \times \mathrm{G}\left(V_{\mathrm{G}}\right)$. We have also directly measured photocurrent and found similar results. Photovoltage and photocurrent measurements were carried out using a lock-in amplifier, modulating the laser intensity at $2 \mathrm{kHz}$ with an optical chopper. The laser wavelength was $650 \mathrm{~nm}$, and the laser power on the sample $160 \mathrm{nW}$. Four different devices were measured, showing similar behaviour.

Received 20 April 2010; accepted 7 July 2010; published online 22 August 2010

\section{References}

1. Chakraborty, T. \& Pietiläinen, P. The Quantum Hall Effects (Springer, 1995).

2. McCormick, K. L., Woodside, M. T., Huang, M., Wu, M. \& McEuen, P. L. Scanned potential microscopy of edge and bulk currents in the quantum Hall regime. Phys. Rev. B 59, 4654-4657 (1999).

3. Ahlswede, E., Weitz, P., Weis, J., von Klitzing, K. \& Eberl, K. Hall potential profiles in the quantum Hall regime measured by a scanning force microscope. Phys. B Condens. Matter 298, 562-566 (2001).

4. Novoselov, K. S. et al. Two-dimensional gas of massless Dirac fermions in graphene. Nature 438, 197-200 (2005).

5. Zhang, Y., Tan, J. W., Stormer, H. L. \& Kim, P. Experimental observation of the quantum Hall effect and Berry's phase in graphene. Nature 438, 201-204 (2005).

6. Williams, J. R., DiCarlo, L. \& Marcus, C. M. Quantum Hall effect in a gate-controlled p-n junction of graphene. Science 317, 638-641 (2007).

7. Ozyilmaz, B. et al. Electronic transport and quantum Hall effect in bipolar graphene p-n-p junctions. Phys. Rev. Lett. 99, 166804 (2007).

8. Ki, D. K., Jo, S. \& Lee, H. J. Observation of chiral quantum-Hall edge states in graphene. Appl. Phys. Lett. 94, 162113 (2009).

9. Buttiker, M. Absence of backscattering in the quantum Hall-effect in multiprobe conductors. Phys. Rev. B 38, 9375-9389 (1988).

10. Chklovskii, D. B., Shklovskii, B. I. \& Glazman, L. I. Electrostatics of edge channels. Phys. Rev. B 46, 4026-4034 (1992).

11. Martin, J. et al. The nature of localization in graphene under quantum Hall conditions. Nature Phys. 5, 669-674 (2009).

12. Connolly, M. R. et al. Scanning gate microscopy of current-annealed single layer graphene. Appl. Phys. Lett. 96, 113501-113503.

13. Williams, J. R., Abanin, D. A., DiCarlo, L., Levitov, L. S. \& Marcus, C. M. Quantum Hall conductance of two-terminal graphene devices. Phys. Rev. B 80, 045408 (2009).

14. Martin, J. et al. Observation of electron-hole puddles in graphene using a scanning single-electron transistor. Nature Phys. 4, 144-148 (2008).

15. Abanin, D. A. \& Levitov, L. S. Conformal invariance and shape-dependent conductance of graphene samples. Phys. Rev. B 78, 035416 (2008).

16. Lee, E. J. H., Balasubramanian, K., Weitz, R.T., Burghard, M. \& Kern, K. Contact and edge effects in graphene devices. Nature Nanotech. 3, 486-490 (2008).

17. Xia, F. N. et al. Photocurrent imaging and efficient photon detection in a graphene transistor. Nano Lett. 9, 1039-1044 (2009).

18. Mueller, T., Xia, F., Freitag, M., Tsang, J. \& Avouris, P. Role of contacts in graphene transistors: A scanning photocurrent study. Phys. Rev. B 79, 245430 (2009). 
19. Park, J., Ahn, Y. H. \& Ruiz-Vargas, C. Imaging of photocurrent generation and collection in single-layer graphene. Nano Lett. 9, 1742-1746 (2009).

20. Xu, X. D., Gabor, N. M., Alden, J. S., van der Zande, A. M. \& McEuen, P. L. Photo-thermoelectric effect at a graphene interface junction. Nano Lett. 10, 562-566 (2010).

21. Vanharen, R. J. F., Delange, W., Blom, F. A. P. \& Wolter, J. H. Imaging of edge channels in the integer quantum Hall regime by the lateral photoelectric effect. Phys. Rev. B 52, 5760-5766 (1995).

22. Shashkin, A. A. et al. Hall photovoltage imaging of the edge of a quantum Hall device. Phys. Rev. Lett. 79, 5114-5117 (1997).

23. Vanharen, R. J. F., Blom, F. A. P. \& Wolter, J. H. Direct observation of edge channels in the integer quantum Hall regime. Phys. Rev. Lett. 74, 1198-1201 (1995).

24. Ioannou, D. E. \& Dimitriadis, C. A. A SEM-EBIC minority-carrier diffusion-length measurement technique. IEEE Trans. Electr. Devices 29, $445-450$ (1982).

25. Kelzenberg, M. D. et al. Photovoltaic measurements in single-nanowire silicon solar cells. Nano Lett. 8, 710-714 (2008).

26. Chklovskii, D. B., Matveev, K. A. \& Shklovskii, B. I. Ballistic conductance of interacting electrons in the quantum Hall regime. Phys. Rev. B 47, 12605-12617 (1993).

27. Kicin, S. et al. Local backscattering in the quantum Hall regime. Phys. Rev. B 70, $205302(2004)$
28. Bolotin, K. I., Sikes, K. J., Hone, J., Stormer, H. L. \& Kim, P.

Temperature-dependent transport in suspended graphene. Phys. Rev. Lett. 101, 096802 (2008).

29. Novoselov, K. S. et al. Electric field effect in atomically thin carbon films. Science 306, 666-669 (2004).

30. Novoselov, K. S. et al. Two-dimensional atomic crystals. Proc. Natl Acad. Sci. USA 102, 10451-10453 (2005).

\section{Acknowledgements}

Work carried out under the auspices of the US Department of Energy under contract DE-AC02-98CH1-886. Y.Z. acknowledges financial support from NSF contract DMR-0705131.

\section{Author contributions}

P.S., E.S. and G.N. designed the study; G.N. carried out the experiments and analysed the data; Y.Z. and L.Z. provided samples; G.N., P.S. and E.S. wrote the paper; all authors commented on the manuscript.

\section{Additional information}

The authors declare no competing financial interests. Supplementary information accompanies this paper on www.nature.com/naturephysics. Reprints and permissions information is available online at http://npg.nature.com/reprintsandpermissions. Correspondence and requests for materials should be addressed to P.S. 